\title{
An Overview of Systematic Reviews of Shenmai Injection for Healthcare
}

\author{
Ling-yan Lu, ${ }^{1}$ Guo-qing Zheng, ${ }^{2}$ and Yan Wang ${ }^{1}$ \\ ${ }^{1}$ Department of Cardiology, The Second Affiliated Hospital of Wenzhou Medical University, Wenzhou 325027, China \\ ${ }^{2}$ Department of Neurology, The Second Affiliated Hospital of Wenzhou Medical University, Wenzhou 325027, China
}

Correspondence should be addressed to Guo-qing Zheng; gq_zheng@sohu.com and Yan Wang; wywzchina@sina.com

Received 15 November 2013; Accepted 2 January 2014; Published 12 February 2014

Academic Editor: Byung-Cheul Shin

Copyright (c) 2014 Ling-yan Lu et al. This is an open access article distributed under the Creative Commons Attribution License, which permits unrestricted use, distribution, and reproduction in any medium, provided the original work is properly cited.

\begin{abstract}
Shenmai injection (SMI) is widely applied in clinical practice as an organ protector. This overview is to evaluate the current evidence from systematic reviews (SRs) of SMI for healthcare. The literature searches were carried out in 6 databases without language restrictions until December 2012. The quality of the primary studies from the respective SRs was evaluated by using Jadad score. The overview quality assessment questionnaire (OQAQ) was used to evaluate the methodological quality of all included SRs. Twenty eligible SRs were identified. They reported a wide range of conditions, including SMI for cardio/cerebrovascular diseases, viral myocarditis, tumor chemotherapy, and adverse drug reactions. Most of the primary studies were of good quality only in 1 SR of non-small-cell lung cancer. According to the OQAQ scores, the quality of included SRs was variable and six reviews were of high quality with a score of 5 points. Two SRs showed that SMI had low adverse drug reaction occurrence. In conclusion, there is mixed evidence to support efficacy of SMI for an adjunct therapy to tumor chemotherapy and premature evidence for the use of SMI for cardio/cerebrovascular disorders and viral myocarditis. SMI seems generally safe for clinical application. Further large sample-size and well-designed RCTs are needed.
\end{abstract}

\section{Introduction}

Shenmai injection (SMI) is derived from the famous traditional Chinese herbal prescription Shendong yin, whose formulation was first recorded in Zhengyin Maizhi (Pattern, Cause, Pulse, and Treatment) by Jing-Ming Qin in $1702 \mathrm{AD}$ [1]. Shendong Yin consists of ginseng (Radix Ginseng, the root of Panax ginseng C.A. Mey., Araliaceae) and dwarf lilyturf tuber (Radix Ophiopogonis, the tuber of Ophiopogon japonicus (Thunb.) Ker-Gawl., Liliaceae) [1]. In modern time, Shendong Yin is still widely used in clinical practice in many countries such as China, Korea, Japan, and Singapore $[2,3]$. According to the theory of traditional Chinese medicine, Shendong Yin has the function of invigorating qi for relieving desertion, nourishing Yin, and replenishing bodily fluids [1, 4]. Ginsenosides and ophiopogonis, isolated from ginseng and dwarf lilyturf tuber, were regarded as the principal constituents responsible for the pharmacological activities [5]. Using Chinese herbal medicine as injections is an innovation that has been proved to be effective in extensive clinical use in Mainland China. SMI was developed as a traditional Chinese patent injection since 1995. Various pharmacological studies indicated that SMI has immunomodulatory effects against tissue damage by suppressing the release of tumor necrosis factor alpha (TNF- $\alpha$ ), nitric oxide (NO), and other inflammatory products from macrophages, and it also has protective effects of dilating the coronary arteries, increasing blood supply, improving microcirculation, and eliminating surplus free radicals [6]. Because of its effectiveness, nowadays SMI has become a famous Chinese patent injection and has been widely applied in clinical practice as an organ protector $[3,6]$.

Evidence-based medicine (EBM) is a strategy for the critical evaluation and uniform comparison of clinical trial data with conclusions according to predetermined efficacy criteria [7]. Several systematic reviews have been conducted to evaluate SMI for a wide range of conditions in clinical practice, including viral myocarditis, acute myocardial infarction, acute cerebral infarction, tumor (as an adjunct 


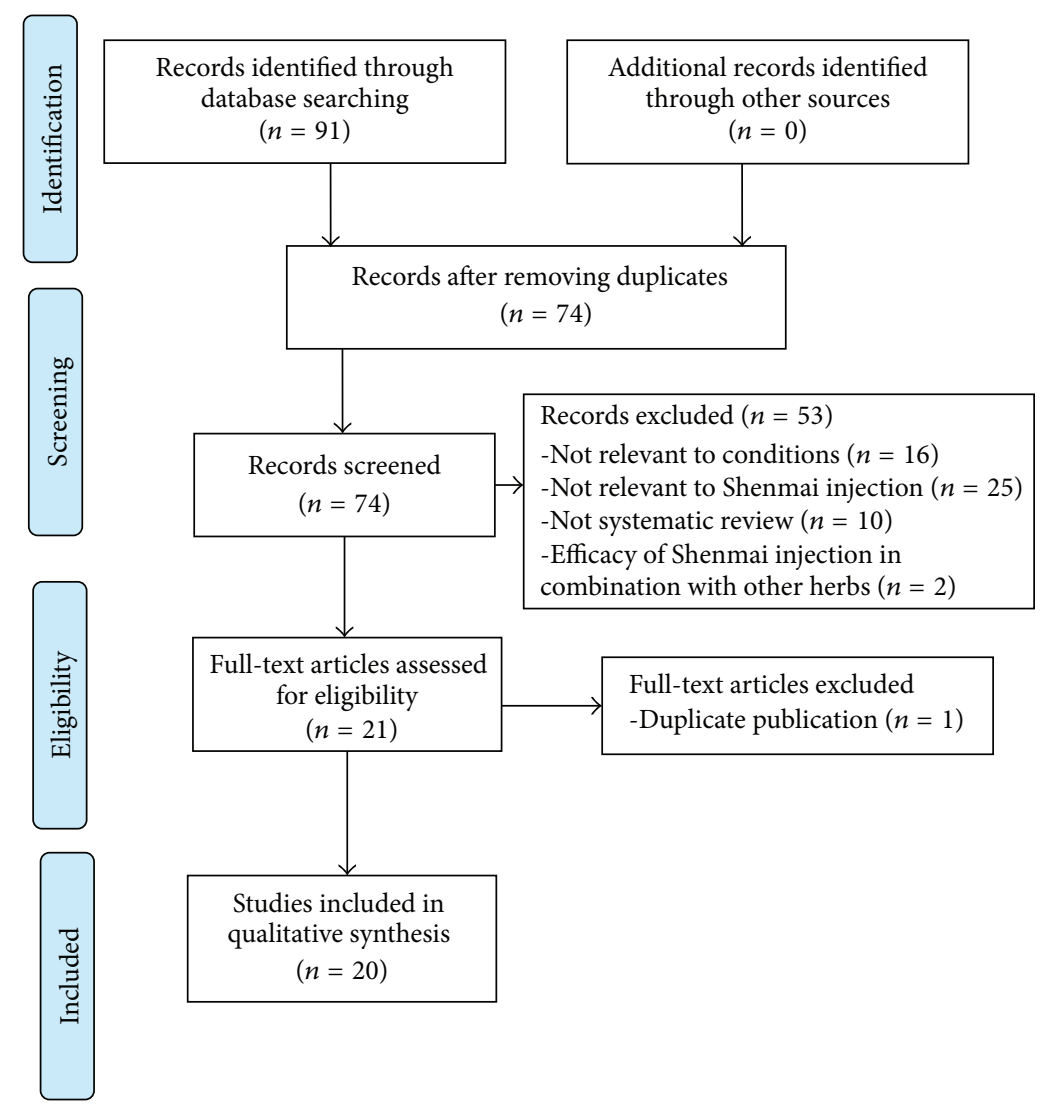

FiguRE 1: PRISMA 2009 Flow Diagram.

therapy to chemotherapy), coronary heart disease, chronic cor pulmonale, dilated cardiomyopathy, and heart failure [825]. However, the results are all positive which basically come from the poor methodological quality. Thus, the objective of this overview is to critically summarize and evaluate systematic reviews of SMI for healthcare.

\section{Methods}

2.1. Eligibility Criteria. Systematic review is defined as an exhaustive review of the literature addressing a clearly defined question, which uses a systematic and explicit methodology to identify, select, and critically evaluate all the relevant studies and collect and analyse the data emerging from the studies included in it [26]. Systematic reviews must include an explicit and repeatable methods section describing the search strategy and explicit inclusion/exclusion criteria [27]. To be considered, the systematic review has to be concerned specifically with the effectiveness of SMI and must include evidence from at least two randomized controlled trials (RCTs). Systematic reviews evaluating SMI together with other Chinese herbal medicine (CHM) and without separate evaluation of the individual drug were excluded. Reviews, comments, and overviews without a systematic methods section were excluded.

2.2. Literature Search. Electronic literature search was carried out in the following databases up to December 31, 2012 without language restrictions: Pubmed, EMBASE, Chinese Hospital Knowledge Database (CHKD), China National Knowledge Infrastructure, VIP Journals Database, and Wanfang Med Online Database (Figure 1). The keywords used in the search were "systematicreview OR meta-analysis" AND "Shenmai." Chinese database was searched using the above search terms in Chinese accordingly.

2.3. Study Selection and Data Collection Process. Data were extracted and evaluated independently by 2 authors using predefined criteria. Disagreements were resolved through consultation with corresponding author. The assessments of the quality of the primary studies which were evaluated by using Jadad score were adopted from the systematic reviews, respectively.

2.4. The Overview Quality Assessment Questionnaire. The overview quality assessment questionnaire (OQAQ) [28-30] which consists of 10 questions was used to evaluate the methodological quality of all included systematic reviews. Questions 1 to 9, which were answered with "adequate," "inadequate," or "not mentioned," addressed the 5 methodological aspects of systematic reviews including search strategy, study selection, validity assessment, data analysis, and inferences. The final question 10 is the overall scientific quality of the review article graded on a 7-point scale. A score of three or less was considered as indicative of extensive or major flaws 
and a score of 5 or more as suggesting minor or minimal flaws. Two authors assess the OQAQ independently. We settled any discrepancies by discussion or consulting with corresponding author.

\section{Results}

3.1. Description of the Screening Process. Our searches generated 91 potentially relevant articles. After removal of duplicates, 74 records remained. Through screening titles and abstracts, we excluded 53 papers with at least one of following reasons: (1) not relevant to conditions; (2) not relevant to SMI; (3) not systematic review; and (4) efficacy of SMI in combination with other CHM. After full-text evaluation on the remaining 21 articles, 1 article was excluded for duplicate publication [31]. Ultimately, 20 eligible studies were selected in present study. Of the 20 eligible studies, two systematic reviews were specifically focused on the adverse drug reactions (ADRs) of SMI [32,33], which we would analysis in a separate part. The screening process is summarized in a flow diagram (Figure 1).

3.2. Study Characteristics. Two systematic reviews were published in English [24, 33] and 18 others in Chinese from 2005 to 2012. The first authors of all included trials were from China and were affiliated to academic institutions. They reported a wide range of conditions, including viral myocarditis $(n=4)$, acute myocardial infarction $(n=$ $3)$, acute cerebral infarction $(n=3)$, tumor (SMI as an adjunct therapy to chemotherapy) $(n=2)$, coronary heart disease $(n=3)$, chronic cor pulmonale $(n=1)$, dilated cardiomyopathy $(n=1)$, and heart failure $(n=1)$. The systematic reviews were based on 6 to 33 primary studies. Most of the primary studies were of good quality only in 1 systematic review [17], while almost all the primary studies were of poor quality or $\mathrm{C}$ grade in the other 17 systematic reviews according to the Jadad score. All systematic reviews applied a meta-analytic approach. Key data of the included systematic reviews are summarized in Table 1.

3.3. Assessing the Quality of Systematic Reviews. According to the OQAQ scores, the quality of these reviews were varied. Six systematic reviews included were considered to having minor or minimal flaws. that is, scoring 5 points on the OQAQ $[8,9,14,17,21,24]$. The other 12 systematic reviews were mostly of poor quality $[10-13,15,16,18-20,22,23,25]$. Among them, 3 systematic reviews scored 4 points $[19,22,23]$, and the remaining 9 trials which had major flaws scored equal to or less than 3 points $[10-13,15,16,18,20,25]$. The details of the assessment of the quality of systematic reviews are listed in Table 2.

3.4. Effectiveness. All of the systematic reviews reached positive conclusion (Table 1).

3.5. Adverse Drug Effects. There are 2 reviews addressing the adverse drug effects of SMI $[32,33]$. One review was published in 2003 and only found 16 cases which were grouped into 4 main categories: skin lesions $(n=1)$, anaphylactic shock $(n=5)$, phlebitis $(n=1)$, cardiovascular and cerebrovascular diseases $(n=7)$, and other adverse effects $(n=2)$ [32]. The other review was carried out in 2010 in which they collected relevant information such as gender, age, allergic history, primary diseases treated in ADR cases, types, occurrence times, severity of ADRs, and menstruum and compatibility of SMI. Of the 822 total reported ADR cases, 246 ADR cases resulted from 181 ADR reports and 146 described a total of 576 ADR cases in 1828 clinical studies [33]. This systematic review found that the most commonly affected age group was 40 to 69 . There are 36 cases which were described as having an allergic history. The diseases being treated in ADR cases were principally heart failure and coronary artery heart disease. Thirty-eight of the 246 ADR cases in ADR reports described anaphylactic shock, while the most common ADR reported in clinical studies was headache or dizziness. In classification of ADR severity for SMI, 38 cases, 48 cases, 99 cases, and 637 cases were class I, class II, class III, and class IV, respectively, and there was no death. All in all, this systematic review indicated that SMI had low ADR occurrence, although some potential factors such as irrational compatibility and dosages may lead to a high risk of ADR.

\section{Discussions}

4.1. Summary of Evidence. This overview indicated that a remarkable multitude of systematic reviews of SMI for many types of diseases has emerged between 2003 and 2012, suggesting that the interests of the public and the medical profession in the use of SMI for healthcare have grown considerably in recent years. SMI is commonly used in the cardiocerebrovascular area, in viral myocarditis, and in an adjunct therapy to tumor chemotherapy. There is mixed evidence to support efficacy of SMI for an adjunct therapy to tumor chemotherapy and premature evidence for the use of SMI for cardiocerebrovascular disorders and viral myocarditis, whereas SMI treatment was generally safe for healthcare.

4.2. Limitations. The OQAQ scale was selected to assess various aspects of the methodological quality of systematic reviews. However, only $6 / 18$ scoring 5 or above indicates that the study has minimal flaws. Second, this review rest with inherent limitations in the primary studies. The only trial [17] is SMI as an adjunct therapy for patients with NSCLC based on more than half of the high quality of the included primary studies. Third, all the studies met the criteria coming from China was another weakness that potentially limited the generalizability of the findings. SMI belongs to Chinese traditional medicine patent injection and is only available in the domestic market. Thus, relevant researches are limited to Chinese researchers. Fourth, All of the systematic reviews reached positive conclusion. Several groups have shown that nearly $100 \%$ of all Chinese trials reported positive results [34]. Thus, the evidences have to be interpreted with caution. At last, due to the remarkable multitude of databases, the literature search may not be whole and some important data 


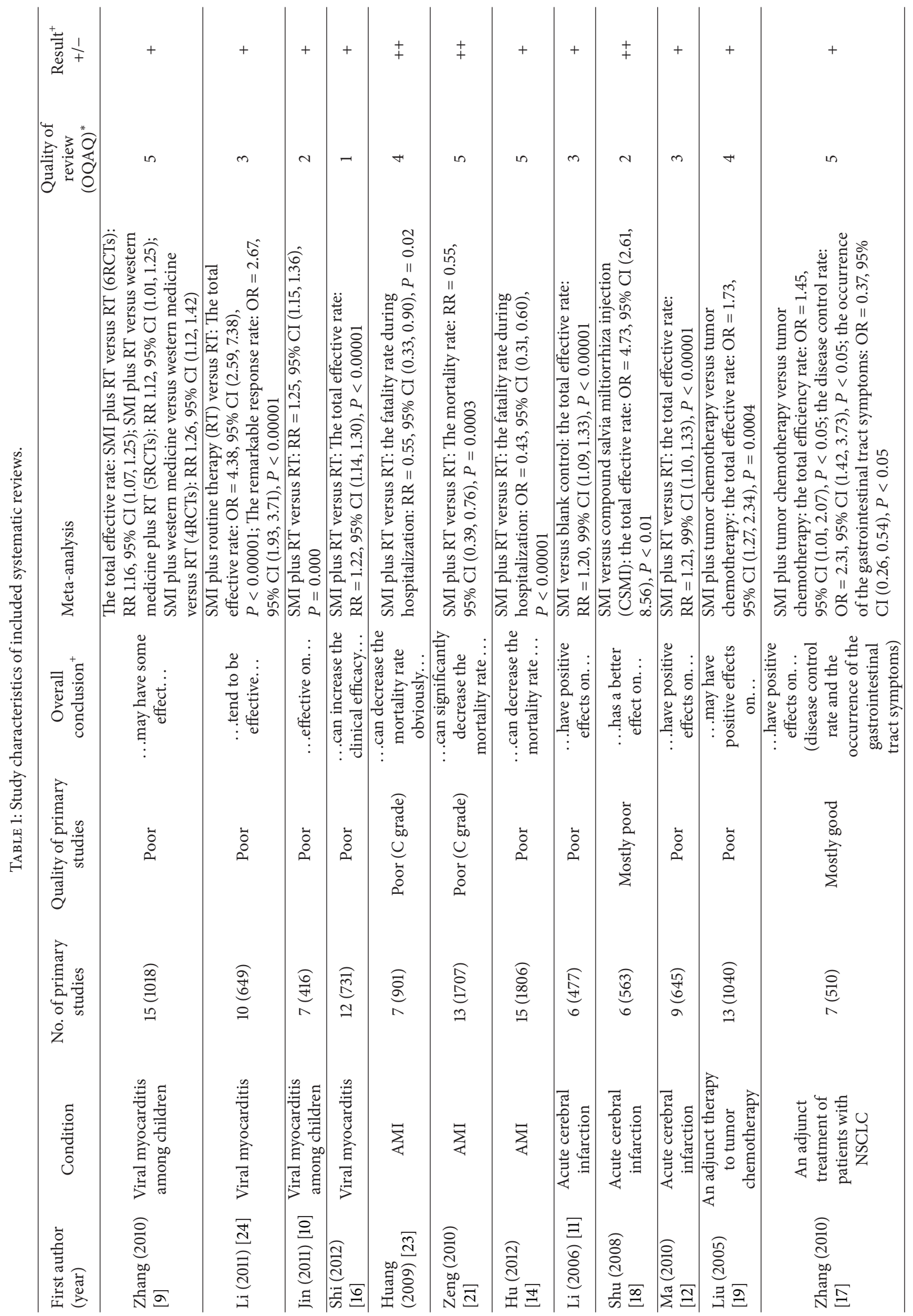




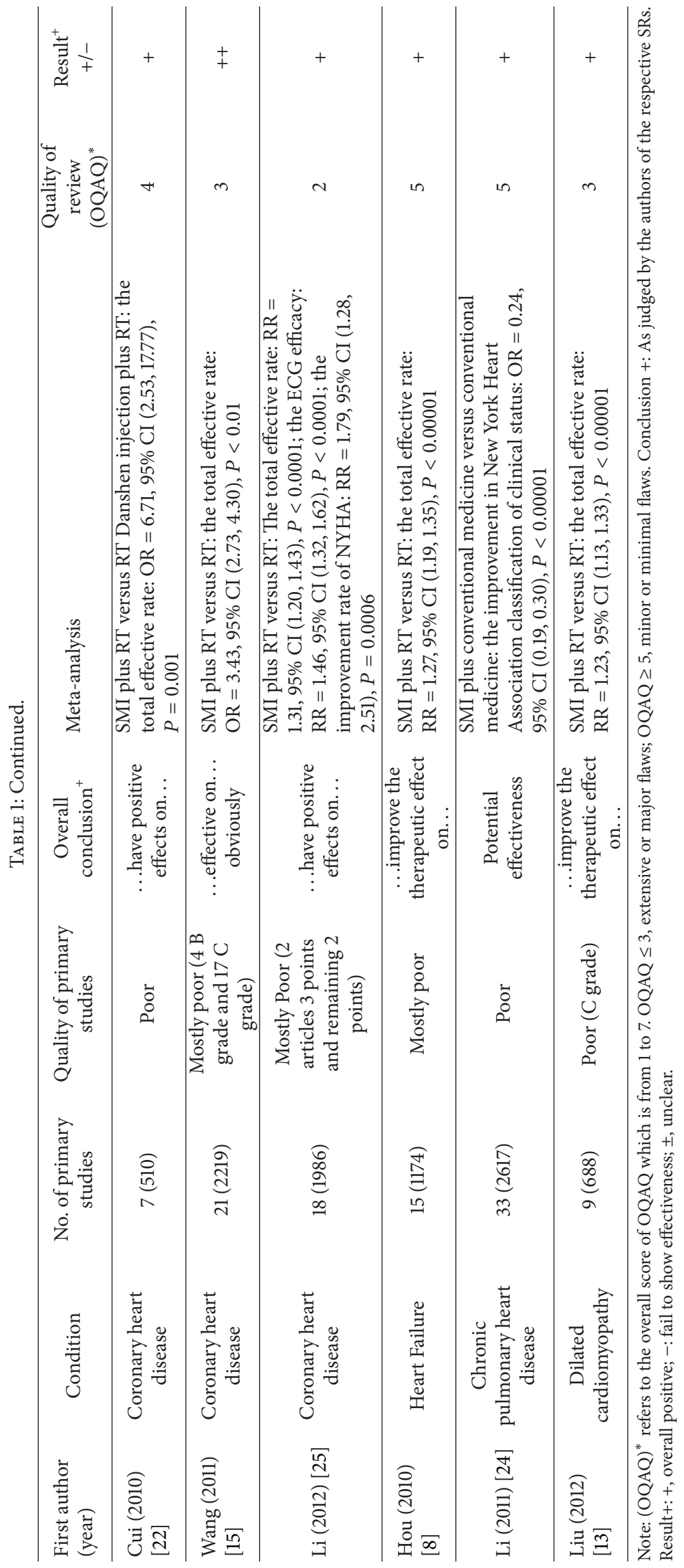




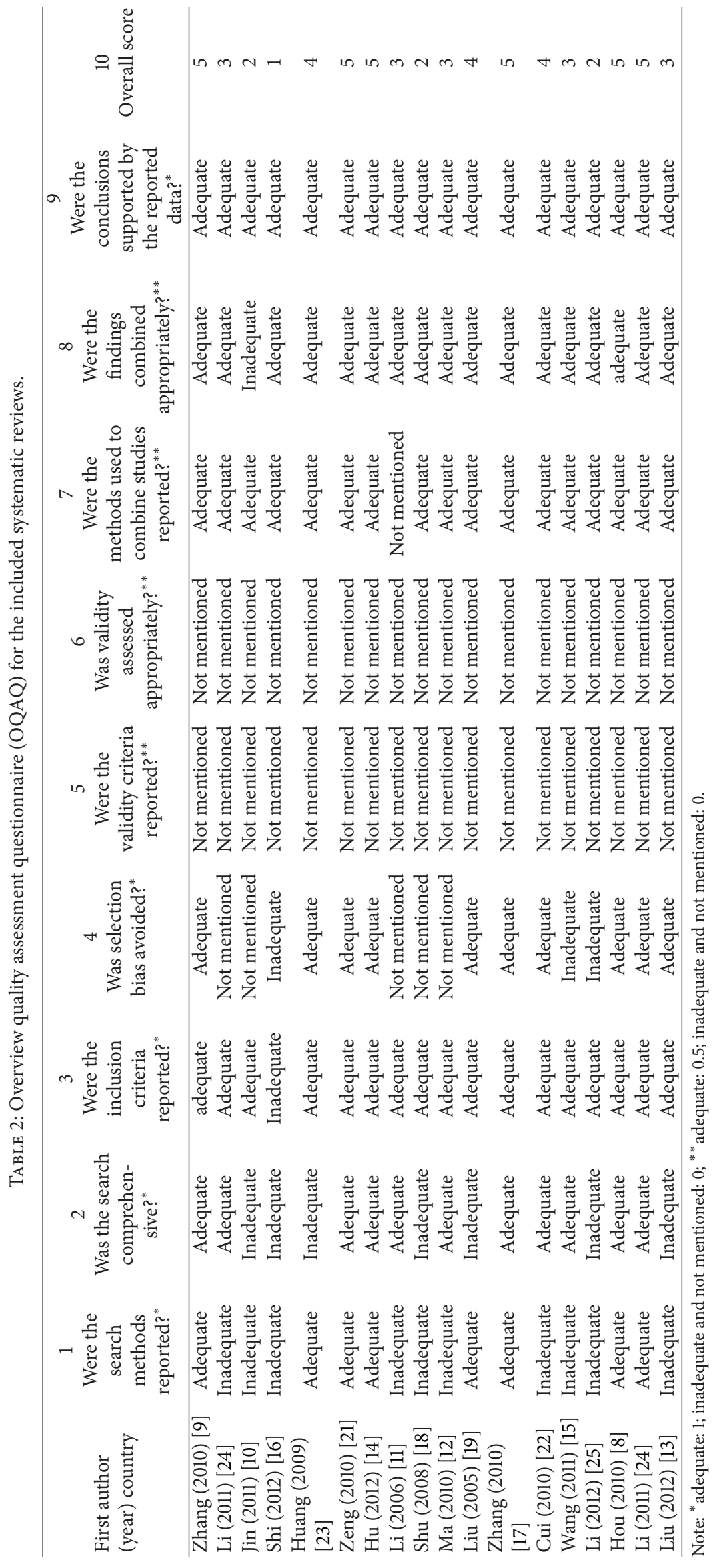


may be lost. This could have a potential influence on the outcomes.

4.3. Implication of Practice. This review revealed mixed evidence in support of the use of SMI for an adjunct therapy to tumor chemotherapy and premature evidence for the use of SMI for the cardiocerebrovascular area and viral myocarditis. Additionally, SMI appeared to be well tolerated in almost all included patients. However, it should be remembered that a lack of scientific evidence does not necessarily mean that the treatment is ineffective [35]. Since SMI is not available in the western countries, we suggested that oral administration of Shendong yin could be an alternative option for healthcare.

4.4. Cancer. There are two studies about SMI as an adjunct therapy for patients with cancer. One contains 13 primary studies which included in 1040 patients with all types of cancer [19] and the other contains 7 primary studies which included in 510 patients specifically with NSCLC [17]. As for the former, although it has rigorous and positive studies, the current evidence is insufficient for support of the effectiveness of SMI as an adjunct therapy of cancer due to the poor methodological quality in the primary studies. However, the evidence for NSCLC is supported by probable evidence for routinely clinical use because of its more than half high methodological quality of the included primary studies and scoring 5 points on the OQAQ. In pharmacological studies, the antitumor effects of a ginsenoside Rg3-fortified red ginseng preparation (Rg3-RGP) were investigated in human NSCLC (H460) cells using in vitro cytotoxicity assay and in vivo nude mouse xenograft model, suggesting that Rg3-RGP could exert antitumor activities through indirect immunomodulatory actions, without causing adverse effects as caused by doxorubicin [36].

4.5. Cardiocerebrovascular Diseases. Cardiocerebrovascular diseases are the leading cause of death worldwide and the first cause of acquired disability, and their costs both direct and indirect are astronomic. At least 12 SRs have been published in this area, suggesting that SMI is mainly and widely used in cardio/cerebrovascular diseases, including acute myocardial infarction $(n=3)$, acute cerebral infarction $(n=3)$, coronary heart disease $(n=3)$, chronic cor pulmonale $(n=1)$, dilated cardiomyopathy $(n=1)$, and heart failure $(n=1)$. Despite this wealth of clinical use, there are insufficient evidence to conclude efficacy of SMI for cardiocerebrovascular diseases because of low methodological quality of the primary included trials.

4.6. Viral Myocarditis. Viral myocarditis is one of the most challenging diseases to diagnose and treat in cardiology and has been commonly associated with a viral infection. Treatment of acute viral myocarditis is still an integrative and aggressive supportive care, except for giant cell myocarditis where immunotherapy has been shown to improve survival [37]. Currently, many patients turn to herbal medicine, a form of the main part of traditional Chinese medicine, when conventional medicine fails them or they believe strongly in the effectiveness of complementary medicine. An updated systematic review which was recently published by Liu et al. [38] indicated that numerous clinical trials have been conducted to investigate the efficacy and safety of herbal medicines for viral myocarditis, suggesting that there is no evidence of benefit of herbal medicine on all-cause mortality, and some herbal medicines may lead to improvement of ventricular premature beat, electrocardiogram, levels of myocardial enzymes, and cardiac function in viral myocarditis, alleviating myocardial damage, and improving the effect of treatment of viral myocarditis. In the present study, four SRs were selected for viral myocarditis. Only one SR is rigorous and based on a sufficiently large number of primary studies, which is scored 5 points. However, its primary studies are of poor methodological quality. Therefore, its positive conclusion should treated cautiously.

4.7. Implication of Research. A number of implications for research arise from this review. First, improvement in the methodological quality of primary RCTs is crucial for future clinical studies. We recommend that some specific guidelines such as the CONSORT 2010 statement [39] and guidelines for RCTs investigating CHM [40] and CONSORT for TCM [41] should be used as a combined guideline when designing and reporting RCTs for CHM. Second, SRs must be of high quality. The overall quality of present included SRs is not so good. Thus, there is room for further improvement of methodological quality of SRs. Reports of SRs and metaanalyses should use the PRISMA statement [42] as a guide and encourage the prospective registration of SRs. Third, the safety of herbal patent injection has become a major concern to both national health authorities and the general public. The quantity of published literatures ranked SMI the ninth in ADRs of 33 varieties of CHM Injections on the 2004 edition of National Essential Drugs List (2004 edition) of China [43]. In the present overview, SMI appears to be well tolerated. While reporting of $\mathrm{ADR}$ following $\mathrm{CHM}$ injection is becoming more and more common, the reporting quality is of concern. A standard reporting format for ADR has been developed [44] and we suggested that improvement of reporting of adverse events and ADRs of SMI may follow up. Fourth, SMI is widely used in the treatment of various disorders and seems generally safe. Although the current evidence is insufficient to support routine use of SMI, it is a promising candidate for further clinical trial of various disorders, especially an adjunct therapy to tumor chemotherapy, cardiocerebrovascular area, and viral myocarditis.

\section{Conclusions}

The interests of the public and the medical profession in the use of SMI for healthcare have grown considerably in recent years. There is mixed evidence to support efficacy of SMI for an adjunct therapy to tumor chemotherapy and premature evidence for the use of SMI for cardiocerebrovascular disorders and viral myocarditis. In addition, SMI seems generally safe for clinical application. Further large sample-size and well-designed RCTs are needed. 


\section{Conflict of Interests}

The authors declare that there is no conflict of interests regarding the publication of this paper.

\section{Acknowledgment}

This project was supported by the funding of the young and middle-aged university discipline leaders of Zhejiang province, China (2013277).

\section{References}

[1] C. Y. Qin, Zhengyin Maizhi (Pattern, Cause, Pulse, and Treatment), Ancient Books of TCM, Beijing, China, 2000.

[2] C.-H. Xia, J.-G. Sun, G.-J. Wang et al., "Herb-drug interactions: in vivo and in vitro effect of shenmai injection, a herbal preparation, on the Metabolic activities of hepatic cytochrome P450 3A1/2, 2C6, 1A2, and 2E1 in rats," Planta Medica, vol. 76, no. 3, pp. 245-250, 2010.

[3] L. Wang, W. Wang, X. Zhao et al., "Effect of Shenmai injection, a traditional Chinese medicine, on pulmonary dysfunction after tourniquet-induced limb ischemia-reperfusion," Journal of Trauma, vol. 71, no. 4, pp. 893-897, 2011.

[4] L.-M. Zhao, L.-J. Ma, L.-X. Zhang, and J.-Z. Wu, "Shenmai injection inhibiting the extracellular signal regulated kinaseinduced human airway smooth muscle proliferation in asthma," Chinese Journal of Integrative Medicine, vol. 16, no. 4, pp. 331336, 2010.

[5] C. Xia, G. Wang, J. Sun et al., "Simultaneous determination of ginsenoside Rgl, Re, Rd, Rbl and ophiopogonin D in rat plasma by liquid chromatography/electrospray ionization mass spectrometric method and its application to pharmacokinetic study of 'SHENMAI' injection," Journal of Chromatography B, vol. 862, no. 1-2, pp. 72-78, 2008.

[6] Y. Bai, H.-X. Yao, M.-L. Hu et al., "Effects of shenmai injection on pulmonary aquaporin 1 in rats following traumatic brain injury," Chinese Medical Journal, vol. 124, no. 3, pp. 457-460, 2011.

[7] S. H. Fox, R. Katzenschlager, S.-Y. Lim et al., "The movement disorder society evidence-based medicine review update: treatments for the motor symptoms of Parkinson's disease," Movement Disorders, vol. 26, supplement 3, pp. S2-S41, 2011.

[8] Y.-Z. Hou, J.-Y. Mao, X.-L. Wang, C.-X. Liu, and C. Zhang, "Shenmai injection in heart failure patients: a systematic review and Meta-analysis," Chinese Journal of Evidence-Based Medicine, vol. 10, no. 8, pp. 939-945, 2010.

[9] L. Zhang, B.-H. Wang, J. Hu, and H.-C. Shang, "Shenmai injection for children with viral myocarditis: a systematic review," Chinese Journal of Evidence-Based Medicine, vol. 10, no. 8, pp. 939-945, 2010.

[10] X. Y. Jin, "Shenmai injection for viral myocarditis among children: a Meta-analysis," Changzhou Practical Medical, vol. 27, no. 5, pp. 281-285, 2011.

[11] K. J. Li, "Shenmai injection for acute ischemic stroke: a systematic review of randomized controlled trial," Acta Chinese Medicine and Pharmacology, vol. 34, no. 4, pp. 4-7, 2006.

[12] L. H. Ma and K. J. Li, "Shenmai injection for acute ischemic stroke: a Meta-analysis of randomized controlled trial," Liaoning Journal of Tradtional Chinese Medicine, vol. 37, no. 11, pp. 20842085, 2010.
[13] J. G. Liu, "Systematic review on Shenmai Injection treatment of dilated cardiomyopathy," Chinese Traditional Patent Medicine, vol. 34, no. 8, pp. 1456-1461, 2012.

[14] J. Hu, W. Zhang, Y. M. Xie, L. X. Wang, X. L. Nie, and Y. L. Zhang, "Meta-analysis of Shenmai injection treatment for acute myocardial infarction.," China Journal of Chinese Materia Medica, vol. 37, no. 18, pp. 2760-2767, 2012.

[15] Y. Wang, K. F. Ma, X. G. Zhang, Y. Z. Hu, and J. Z. Shentu, "Shenmai Injection in coronary heart disease patients: a systematic review and Meta-analysis," Chinese Journal of Hospital Pharmacy, vol. 31, no. 15, pp. 1314-1317, 2011.

[16] M. Shi and Y. Shen, "Shenmai Injection for treatment of viral myocarditis: a Meta-analysis," Chinese Traditional Patent Medicine, vol. 34, no. 10, pp. 1882-1886, 2012.

[17] W. L. Zhang, T. H. Yan, B. Liu et al., "Systematic reviews and Meta-analysis of Shenmai injection with chemotherapy on treatment of patients with NSCLC," Practical Pharmacy and Clinical Remedies, vol. 14, no. 2, pp. 95-101, 2011.

[18] J. Z. Shu and Q. R. Wang, "Meta analysis of the treatment for acute cerebral inf arction with Shenmai injection and compound salvia miltiorrhiza injection," Modern Journal of Integrated Traditional Chinese and Western Medicines, vol. 17, no. 7, pp. 973-976, 2008.

[19] X.-Y. Liu, S.-L. Lai, X.-F. Guo, and Y.-R. Lao, "Shen-Mai injection as an adjunct therapy to tumor chemotherapy-a systematic review," Chinese Journal of Evidence-Based Medicine, vol. 5, no. 1, pp. 22-28, 2005.

[20] X. Li and Y. Wu, "Shenmai Injection for auxiliary treatment of viral myocarditis: a Meta-analysis," Journal of Chinese Medicinal Materials, vol. 35, no. 7, pp. 1185-1188, 2012.

[21] Y. J. Zeng, J. Wang, Y. C. Zhou, and M. X. Huang, "Effect of shenmai injection on mortality rate of patients with acute myocardial infarction: a systematic review," Modern Journal of Integrated Traditional Chinese and Western Medicine, vol. 19, no. 28, pp. 3555-3558, 2010.

[22] W. P. Cui, B. Du, R. Qu, G. L. Xu, and L. Qin, "Shenmai Injection for treatment of coronary heart disease: a Metaanalysis," Lishizhen Medicine and Materia Medica Research, vol. 21, no. 11, pp. 2990-2991, 2010.

[23] S. M. Huang, Effect of shenmai injection on the mortality rate and complications of patients with acute myocardial infarction: a Meta-analysis. Southern medical university master level 2006 [bachelor's thesis], 2009.

[24] J.-S. Li, H.-F. Wang, S.-Y. Li, X.-Q. Yu, and Z.-W. Wang, "Shenmai injection for chronic pulmonary heart disease: a systematic review and Meta-analysis," Journal of Alternative and Complementary Medicine, vol. 17, no. 7, pp. 579-587, 2011.

[25] F. D. Li and Y. Shen, "Effect and security of shenmai injection for treatment of coronary heart disease: a Meta-analysis," China Journal of Chinese Materia Medica, vol. 37, no. 23, pp. 3651-3658, 2012.

[26] E. Abalos, G. Carroli, M. E. Mackey, and E. Bergel, Critical appraisal of systematic reviews: the WHO reproductive health library 4WHO/RHR/01.6, The World Health Organization, Geneva, Switzerland, 2001.

[27] E. Ernst, M. S. Lee, and T.-Y. Choi, "Acupuncture for insomnia? An overview of systematic reviews," European Journal of General Practice, vol. 17, no. 2, pp. 116-123, 2011.

[28] K. Al Faleh and M. Al-Omran, "Reporting and methodologic quality of cochrane neonatal review group systematic reviews," BMC Pediatrics, vol. 9, article 38, 2009. 
[29] M. S. Lee and E. Ernst, "Systematic reviews of tai chi: an overview," British Journal of Sports Medicine, vol. 46, pp. 713718, 2012.

[30] A. D. Oxman and G. H. Guyatt, "Validation of an index of the quality of review articles," Journal of Clinical Epidemiology, vol. 44, no. 11, pp. 1271-1278, 1991.

[31] X. Y. Liu, S. L. La, and X. F. Guo, “The synergism and attenuation of ShenMai Injection on tumor chemotherapy: a Systematic Review," in Proceedings of the Memoir of the 2nd International Integrated Traditional and Western Medicine, Traditional Chinese Medicine Tumor Academic Conferences, pp. 621-628, 2005.

[32] B. R. She and Q. H. Yang, "Four kinds of commonly used traditional Chinese medicine injection adverse reaction literature data analysis," Practical Clinical Journal of Integrated Traditional Chinese and Western Medicine, vol. 3, no. 1, pp. 53-54, 2003.

[33] L. Zhang, J. Hu, L. Xiao et al., "Adverse drug reactions of Shenmai injection: a systematic review," Journal of EvidenceBased Medicine, vol. 3, no. 3, pp. 177-182, 2010.

[34] A. Vickers, N. Goyal, R. Harland, and R. Rees, "Do certain countries produce only positive results? A systematic review of controlled trials," Controlled Clinical Trials, vol. 19, no. 2, pp. 159-166, 1998.

[35] V. Kotsirilos, "Complementary and alternative medicine. Part 2-evidence and implications for GPs," Australian family physician, vol. 34, no. 8, pp. 689-691, 2005.

[36] D. Park, D.-K. Bae, J. H. Jeon et al., "Immunopotentiation and antitumor effects of a ginsenoside $\mathrm{Rg} 3$-fortified red ginseng preparation in mice bearing H460 lung cancer cells," Environmental Toxicology and Pharmacology, vol. 31, no. 3, pp. 397-405, 2011.

[37] A. Shauer, I. Gotsman, A. Keren et al., "Acute viral myocarditis: current concepts in diagnosis and treatment," Israel Medical Association Journal, vol. 15, no. 3, pp. 180-185, 2013.

[38] Z. L. Liu, Z. J. Liu, J. P. Liu, and J. S. Kwong, "Herbal medicines for viral myocarditi," Cochrane Database of Systematic Reviews, vol. 8, Article ID CD003711, 2013.

[39] K. F. Schulz, D. G. Altman, and D. Moher, "CONSORT 2010 statement: updated guidelines for reporting parallel group randomised trials," British Medical Journal, vol. 340, article c332, 2010.

[40] A. Flower, C. Witt, J. P. Liu, G. Ulrich-Merzenich, H. Yu, and G. Lewith, "Guidelines for randomised controlled trials investigating Chinese herbal medicine," Journal of Ethnopharmacology, vol. 140, no. 3, pp. 550-554, 2012.

[41] Z. Bian, B. Liu, D. Moher et al., "Consolidated standards of reporting trials (CONSORT) for traditional Chinese medicine: current situation and future development," Frontiers of Medicine in China, vol. 5, no. 2, pp. 171-177, 2011.

[42] A. Liberati, D. G. Altman, J. Tetzlaff et al., "The PRISMA statement for reporting systematic reviews and Meta-analyses of studies that evaluate health care interventions: explanation and elaboration," PLoS Medicine, vol. 6, no. 7, Article ID e1000100, 2009.

[43] L. Wang, Q. Yuan, G. Marshall et al., "Adverse drug reactions and adverse events of 33 varieties of traditional Chinese medicine injections on National Essential medicines List (2004 edition) of China: an overview on published literatures," Journal of Evidence-Based Medicine, vol. 3, no. 2, pp. 95-104, 2010.

[44] Z.-X. Bian, H.-Y. Tian, L. Gao et al., "Improving reporting of adverse events and adverse drug reactions following injections of Chinese materia medica," Journal of Evidence-Based Medicine, vol. 3, no. 1, pp. 5-10, 2010. 


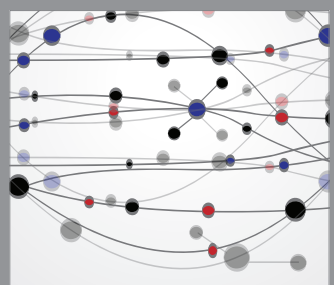

The Scientific World Journal
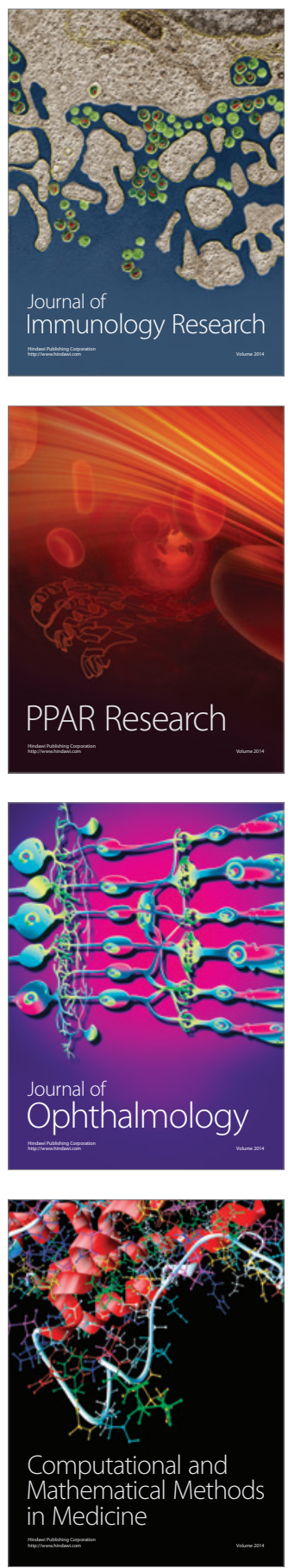

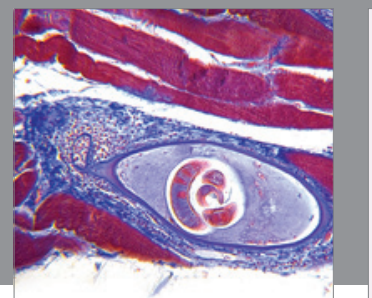

Gastroenterology

Research and Practice
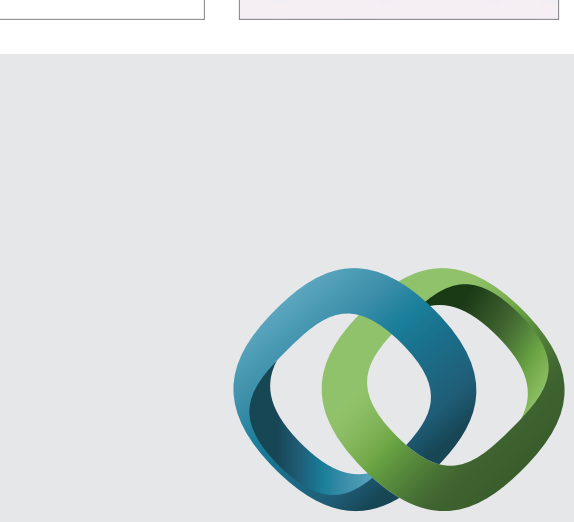

\section{Hindawi}

Submit your manuscripts at

http://www.hindawi.com
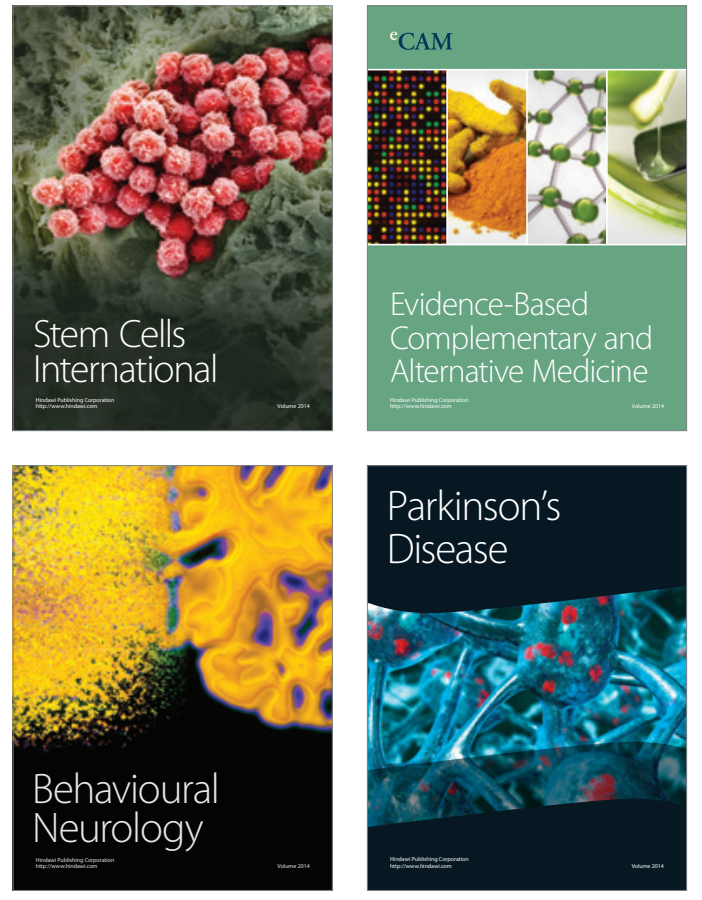
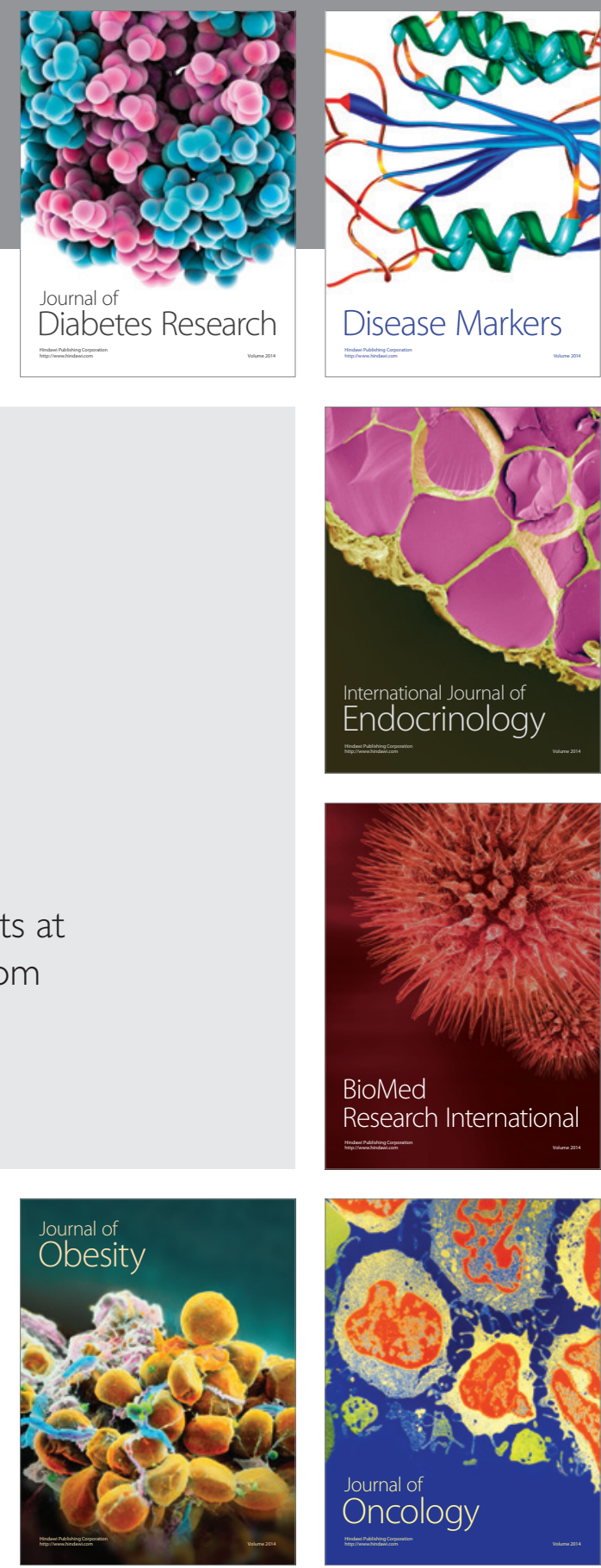

Disease Markers
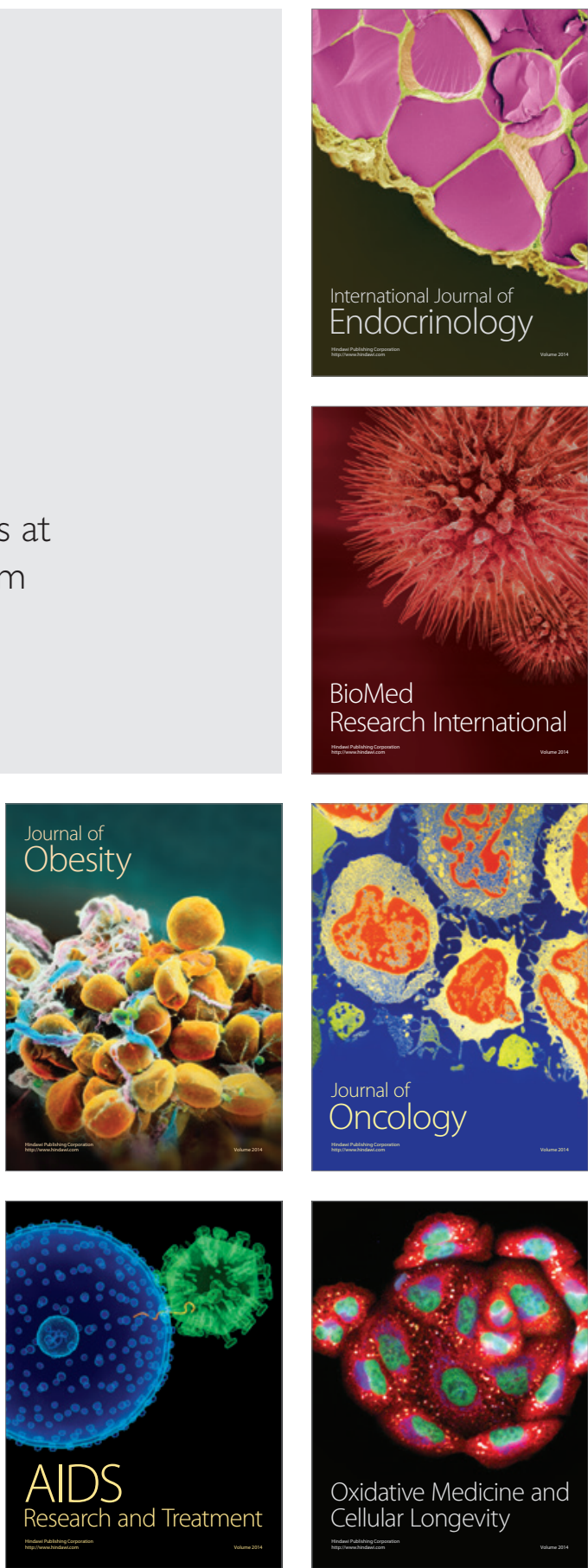